\title{
Phytoplankton Diversity in the World Heritage Site of Indian Sundarbans: An Overview
}

\author{
Atanu Roy ${ }^{1}$, Subrata Trivedi ${ }^{2}$ and Abhijit Mitra ${ }^{3 *}$ \\ ${ }^{1}$ Department of Biotechnology, Techno India University West Bengal, India \\ ${ }^{2}$ Department of Biology, University of Tabuk, Saudi Arabia \\ ${ }^{3}$ Department of Marine Science, University of Calcutta, India
}

*Corresponding author: Abhijit Mitra, Department of Marine Science, University of Calcutta, India

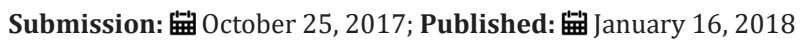

\section{Phytoplankton of Brackish Water System: An Overview}

The pelagic environment of the ocean supports two basic types of marine organisms. One type comprises the plankton, or those organisms whose powers of locomotion are such that they are incapable of making their way against the current and thus are passively transported by currents in the aquatic system and the other type includes the nekton (free swimmers), which are freefloating animals that, in contrast to plankton, are strong enough to swim against currents and are therefore independent of water movements. The category of nekton includes fish, squid and marine mammals.

The word plankton has come from the Greek word "planktos", meaning that which is passively drifting or wandering. Depending upon whether a planktonic organism is a plant or animal, a distinction is made between phytoplankton and zooplankton. Although many planktonic species are of microscopic dimensions, the term is not synonymous with small size as some of the zooplankton includes jellyfish of several meters in diameter. It is not necessary that all plankton are completely passive, most of them are capable of swimming too. Phytoplanktons are free floating tiny floral components that are widely distributed in the marine and estuarine environments. Like land plants, these tiny producers require sunlight, nutrients or fertilizers, carbon dioxide gas and water for growth. The cells of these organisms contain the pigment chlorophyll that traps the solar energy for use in photosynthesis. The photosynthetic process uses the solar radiation to convert carbon dioxide and water into sugars or high energy organic compounds from which the cell forms new materials. The synthesis of organic material by photosynthesis is termed primary production. Since phytoplankton is the dominant producers in the ocean, their role in the marine food chain is of paramount importance. Approximately, 4000 species of marine phytoplankton have been described and new species are continually being added to this total. Phytoplankton exhibit remarkable adaptations to remain in floating condition in the seawater. In fact, all marine phytoplankton tend to stay in the photic zone to utilize the solar radiation for performing the process of photosynthesis.
In order to retard the process of sinking, this group of organisms adopts various mechanisms. These include their small size and general morphology, as the ratio of cell surface area to volume determines frictional drag in the water. Colony or chain formation also increases surface area and slows sinking. Most species carry out ionic regulation, in which the internal concentration of ions is reduced relative to their concentration in seawater. Diatoms also produce and store oil and this metabolic by-product further reduce cell density. In experimental conditions, living cells tend to sink at rates ranging from 0 to $30 \mathrm{~m}$ day- 1 , but dead cells may sink more than twice as fast. In nature, turbulence of surface waters is also an important factor in maintaining phytoplankton near the surface where they receive abundant sunlight.

Phytoplanktons present in the marine and estuarine environments use carbon dioxide for photosynthesis and hence play an important role in maintaining the carbon dioxide budget of the atmosphere. The larger the world's phytoplankton population, the more carbon dioxide gets pulled from the atmosphere. This lowers the average temperature of the atmosphere due to lower volumes of this greenhouse gas. Scientists have found that a given population of phytoplankton can double its numbers in the order of once per day. In other words, phytoplankton responds very rapidly to changes in their environment. Phytoplankton sometimes may cause adverse impact on the marine and estuarine environment. During excessive bloom of phytoplankton, the light energy is intercepted, which could otherwise reach fixed plants like eel grass (Zostera spp.) and kelp. Furthermore, when the phytoplankton eventually die back and break down, an excessive amount of oxygen is required to fuel this process, and hence areas may become deprived of oxygen. Excessive nutrients, and/ or changes in their relative concentrations, may be one factor in a chain of events leading to changes in the species composition of the phytoplankton communities. Increased occurrence of toxic algal blooms may accelerate toxin production. Toxic phytoplankton, when consumed by shellfish or other species can affect the marine food chain, including poisoning of seabirds, mammals and even 
humans. It has been established that phytoplankton naturally contains DMS (dimethyl sulphide), which is released from dead phytoplankton into the atmosphere. This compound can transform into sulphuric acid, which eventually may contribute to acid rain (http://oceanlink.island.net/ask/pollution.html).

\section{Brackish Water Phytoplankton: Major Types}

Nearly all marine plants, whether unicellular or multicellular, even those attached to substrata (sessile) or free floating, pass some part of their life cycle in floating condition as phytoplankton. However, those organisms which always remain planktonic throughout the life cycle are
a) Diatoms,
b) Dinoflagellates,
c) Coccolithophores,
d) Selective species of blue-green algae and
e) Some species of green algae.

\section{Diatoms}

These floating plants are all microscopic in size and are characterised by the presence of shell or frustule. The shell or frustule is composed of translucent silica. The cell wall of diatom has two parts resembling a pillbox bottom and lid. The lid is called the epitheca and the bottom is known as hypotheca. These shells have great importance from the geological point of view and constitute the diatomaceous crust. The diatoms exhibit remarkable varieties and forms and many species possess beautifully sculptured shells. Depending on the nature of valves and pattern of ornamentation in the valve surface, the diatoms are grouped into centric and pennate diatoms. The major differences between these two groups are given in Table 1.

Table 1: Differences between centric and pennate diatoms.

\begin{tabular}{|c|c|c|}
\hline Point & Centric Diatom & Pennate Diatom \\
\hline Cell shape & $\begin{array}{c}\text { Discoid, solenoid or } \\
\text { cylindrical. }\end{array}$ & $\begin{array}{c}\text { Elongated and fusiform, oval, } \\
\text { sigmoid or roughly circular. }\end{array}$ \\
\hline Ornamentation & $\begin{array}{c}\text { Radial in nature i.e., } \\
\text { the arrangement of } \\
\text { markings is radiating } \\
\text { from the centre. }\end{array}$ & $\begin{array}{c}\text { Bilateral in nature i.e., the } \\
\text { arrangement of the markings } \\
\text { is on either side of the apical } \\
\text { (main) axis. }\end{array}$ \\
\hline
\end{tabular}

\section{Dinoflagellates}

These are important producers of the marine environment and rank second in importance in the economy of the sea. Typically, these are unicellular, some are naked while others are armoured with plates of cellulose. The dinoflagellates possess two flagella for locomotion. Several of them are luminescent and produce light. Skeletonema costatum and Coscinodiscus eccentricus are common dinoflagellates in marine and estuarine system.

\section{Coccolithophores}

These are among the smallest category of phytoplankton having a size range between 5 to 20 microns. Some coccolithophores have flagella while others are devoid of them. Their soft bodies are shielded by tiny, calcified circular plates or shields of various designs. These are normally found in the open sea, but their profuse occurrence has been recorded in coastal waters. They form important diet components of filter feeding animals. Examples are Isochrysis galbana and Coccolithus sp.

\section{Blue-Green Algae}

These include both unicellular and multicellular organisms. The blue colour in them is due to the presence of a pigment known as phycocyanin. Of the various organisms belonging to this category, the most important is Trichodesmium erythraeum because in certain seasons of the year its biomass increases greatly resulting in the formation of clumps.

\section{Green Algae}

Microscopic green algae present in the planktonic community largely occur in coastal waters. The green colour in them is due to the presence of chloroplasts. They are widely distributed in the warmer (tropical) seas and only few species are found in the Arctic and Antarctic oceans. Chlorella marina and Chlorella salina are common green algae in estuarine waters.

\section{Phytoplankton Spectrum of Indian Sundarbans}

A field study was undertaken during September, 2017 in the Thakuran River to scan the phytoplankton spectrum in the aquatic phase. A total of 73 species was identified (Table 2) in a salinity range between 12 psu to $18 \mathrm{psu}$. The relatively low salinity compared to average salinity in this region during summer $(\sim 29 \mathrm{psu})$ is due to precipitation and subsequent run-off from the adjacent land masses, which is a characteristics feature of the lower Gangetic delta complex [1,2].

Table 2: List of diatoms identified from the inshore waters of Bay of Bengal.

\begin{tabular}{|c|c|c|}
\hline Sl. No. & Species & Taxonomic Position \\
\hline \multirow{7}{*}{1} & \multirow{7}{*}{ Coscinodiscus eccentricus } & Division: Thallophyta \\
\hline & & Class: Bacillariophyceae \\
\hline & & Order: Centrales \\
\hline & & Sub-order: Cosinodiscineae \\
\hline & & Family: Cosinodisceae \\
\hline & & Genus: Cosinodiscus \\
\hline & & Species: eccentricus \\
\hline
\end{tabular}




\begin{tabular}{|c|c|c|}
\hline \multirow{7}{*}{2} & \multirow{7}{*}{ Coscinodiscus jonesianus } & Division: Thallophyta \\
\hline & & Class: Bacillariophyceae \\
\hline & & Order: Centrales \\
\hline & & Sub-order: Cosinodiscineae \\
\hline & & Family: Cosinodisceae \\
\hline & & Genus: Cosinodiscus \\
\hline & & Species: jonesianus \\
\hline \multirow{7}{*}{3} & \multirow{7}{*}{ Coscinodiscus lineatus } & Division: Thallophyta \\
\hline & & Class: Bacillariophyceae \\
\hline & & Order: Centrales \\
\hline & & Sub-order: Cosinodiscineae \\
\hline & & Family: Cosinodisceae \\
\hline & & Genus: Cosinodiscus \\
\hline & & Species: lineatus \\
\hline \multirow{7}{*}{4} & \multirow{7}{*}{ Coscinodiscus radiates } & Division: Thallophyta \\
\hline & & Class: Bacillariophyceae \\
\hline & & Order: Centrales \\
\hline & & Sub-order: Cosinodiscineae \\
\hline & & Family: Cosinodisceae \\
\hline & & Genus: Cosinodiscus \\
\hline & & Species: radiates \\
\hline \multirow{4}{*}{5} & \multirow{4}{*}{ Coscinodiscus gigas } & Division: Thallophyta \\
\hline & & Class: Bacillariophyceae \\
\hline & & Order: Centrales \\
\hline & & Sub-order: Cosinodiscineae \\
\hline & & Family: Cosinodisceae \\
\hline & & Genus: Cosinodiscus \\
\hline & & Species: gigas \\
\hline \multirow{7}{*}{6} & \multirow{7}{*}{ Coscinodiscus oculus-iridis } & Division: Thallophyta \\
\hline & & Class: Bacillariophyceae \\
\hline & & Order: Centrales \\
\hline & & Sub-order: Cosinodiscineae \\
\hline & & Family: Cosinodisceae \\
\hline & & Genus: Cosinodiscus \\
\hline & & Species: oculus-iridis \\
\hline \multirow{7}{*}{7} & \multirow{7}{*}{ Coscinodiscus concinnus } & Division: Thallophyta \\
\hline & & Class: Bacillariophyceae \\
\hline & & Order: Centrales \\
\hline & & Sub-order: Cosinodiscineae \\
\hline & & Family: Cosinodisceae \\
\hline & & Genus: Cosinodiscus \\
\hline & & Species: concinnus \\
\hline
\end{tabular}




\begin{tabular}{|c|c|c|}
\hline \multirow{7}{*}{8} & \multirow{7}{*}{ Coscinodiscus perforates } & Division: Thallophyta \\
\hline & & Class: Bacillariophyceae \\
\hline & & Order: Centrales \\
\hline & & Sub-order: Cosinodiscineae \\
\hline & & Family: Cosinodisceae \\
\hline & & Genus: Cosinodiscus \\
\hline & & Species: perforates \\
\hline \multirow{7}{*}{9} & \multirow{7}{*}{ Coscinodiscus asteromphalus } & Division: Thallophyta \\
\hline & & Class: Bacillariophyceae \\
\hline & & Order: Centrales \\
\hline & & Sub-order: Cosinodiscineae \\
\hline & & Family: Cosinodisceae \\
\hline & & Genus: Cosinodiscus \\
\hline & & Species: asteromphalus \\
\hline \multirow{7}{*}{10} & \multirow{7}{*}{ Coscinodiscus thorii } & Division: Thallophyta \\
\hline & & Class: Bacillariophyceae \\
\hline & & Order: Centrales \\
\hline & & Sub-order: Cosinodiscineae \\
\hline & & Family: Cosinodisceae \\
\hline & & Genus: Cosinodiscus \\
\hline & & Species: thorii \\
\hline \multirow{7}{*}{11} & \multirow{3}{*}{ Coscinodiscus granii } & Division: Thallophyta \\
\hline & & Class: Bacillariophyceae \\
\hline & & Order: Centrales \\
\hline & & Sub-order: Cosinodiscineae \\
\hline & & Family: Cosinodisceae \\
\hline & & Genus: Cosinodiscus \\
\hline & & Species: granii \\
\hline \multirow{5}{*}{12} & \multirow{5}{*}{ Cyclotella sp } & Division: Thallophyta \\
\hline & & Class: Bacillariophyceae \\
\hline & & Order: Centrales \\
\hline & & Family: Cosinodisceae \\
\hline & & Type: Cyclotella sp \\
\hline \multirow{6}{*}{13} & \multirow{6}{*}{ Cyclotella striata } & Division: Thallophyta \\
\hline & & Class: Bacillariophyceae \\
\hline & & Order: Centrales \\
\hline & & Family: Cosinodisceae \\
\hline & & Genus: Cyclotella \\
\hline & & Species: striata \\
\hline \multirow{6}{*}{14} & \multirow{6}{*}{ Cyclotella stylorum } & Division: Thallophyta \\
\hline & & Class: Bacillariophyceae \\
\hline & & Order: Centrales \\
\hline & & Family: Cosinodisceae \\
\hline & & Genus: Cyclotella \\
\hline & & Species: stylorum \\
\hline
\end{tabular}




\begin{tabular}{|c|c|c|}
\hline \multirow{6}{*}{15} & \multirow{6}{*}{ Thalassiosira subtilis } & Division: Thallophyta \\
\hline & & Class: Bacillariophyceae \\
\hline & & Order: Centrales \\
\hline & & Family: Cosinodisceae \\
\hline & & Genus: Thalassiosira \\
\hline & & Species: subtilis \\
\hline \multirow{5}{*}{16} & \multirow{5}{*}{ Thalassiosira sp. } & Division: Thallophyta \\
\hline & & Class: Bacillariophyceae \\
\hline & & Order: Centrales \\
\hline & & Family: Cosinodisceae \\
\hline & & Type: Thalassiosira sp. \\
\hline \multirow{6}{*}{17} & \multirow{6}{*}{ Thalassiosira decipiens } & Division: Thallophyta \\
\hline & & Class: Bacillariophyceae \\
\hline & & Order: Centrales \\
\hline & & Family: Cosinodisceae \\
\hline & & Genus: Thalassiosira \\
\hline & & Species: decipiens \\
\hline \multirow{6}{*}{18} & \multirow{6}{*}{ Thalassiosira punctigera } & Division: Thallophyta \\
\hline & & Class: Bacillariophyceae \\
\hline & & Order: Centrales \\
\hline & & Family: Cosinodisceae \\
\hline & & Genus: Thalassiosira \\
\hline & & Species: punctigera \\
\hline \multirow{6}{*}{19} & \multirow{6}{*}{ Thalassiosira hyaline } & Division: Thallophyta \\
\hline & & Class: Bacillariophyceae \\
\hline & & Order: Centrales \\
\hline & & Family: Cosinodisceae \\
\hline & & Genus: Thalassiosira \\
\hline & & Species: hyaline \\
\hline \multirow{6}{*}{20} & \multirow{6}{*}{ Thalassiosira eccentric } & Division: Thallophyta \\
\hline & & Class: Bacillariophyceae \\
\hline & & Order: Centrales \\
\hline & & Family: Cosinodisceae \\
\hline & & Genus: Thalassiosira \\
\hline & & Species: eccentric \\
\hline \multirow{6}{*}{21} & \multirow{6}{*}{ Skeletonema costatum } & Division: Thallophyta \\
\hline & & Class: Bacillariophyceae \\
\hline & & Order: Centrales \\
\hline & & Family: Cosinodisceae \\
\hline & & Genus: Skeletonema \\
\hline & & Species: costatum \\
\hline \multirow{6}{*}{22} & \multirow{6}{*}{ Paralia sulcata } & Division: Thallophyta \\
\hline & & Class: Bacillariophyceae \\
\hline & & Order: Centrales \\
\hline & & Family: Cosinodisceae \\
\hline & & Genus: Paralia \\
\hline & & Species: sulcata \\
\hline
\end{tabular}




\begin{tabular}{|c|c|c|}
\hline \multirow{6}{*}{23} & \multirow{6}{*}{ Planktoniella sol } & Division: Thallophyta \\
\hline & & Class: Bacillariophyceae \\
\hline & & Order: Centrales \\
\hline & & Family: Cosinodisceae \\
\hline & & Genus: Planktoniella \\
\hline & & Species: sol \\
\hline \multirow{6}{*}{24} & \multirow{6}{*}{ Planktoniella blanda } & Division: Thallophyta \\
\hline & & Class: Bacillariophyceae \\
\hline & & Order: Centrales \\
\hline & & Family: Cosinodisceae \\
\hline & & Genus: Planktoniella \\
\hline & & Species: blanda \\
\hline \multirow{6}{*}{25} & \multirow{6}{*}{ Rhizosolenia setigera } & Division: Thallophyta \\
\hline & & Class: Bacillariophyceae \\
\hline & & Order: Centrales \\
\hline & & Family: Soleniae \\
\hline & & Genus: Rhizosolenia \\
\hline & & Species: setigera \\
\hline \multirow{6}{*}{26} & \multirow{6}{*}{ Rhizosolenia alata } & Division: Thallophyta \\
\hline & & Class: Bacillariophyceae \\
\hline & & Order: Centrales \\
\hline & & Family: Soleniae \\
\hline & & Genus: Rhizosolenia \\
\hline & & Species: alata \\
\hline \multirow{6}{*}{27} & \multirow{6}{*}{ Rhizosolenia hebetata } & Division: Thallophyta \\
\hline & & Class: Bacillariophyceae \\
\hline & & Order: Centrales \\
\hline & & Family: Soleniae \\
\hline & & Genus: Rhizosolenia \\
\hline & & Species: hebetata \\
\hline \multirow{6}{*}{28} & \multirow{6}{*}{ Rhizosolenia styliformis } & Division: Thallophyta \\
\hline & & Class: Bacillariophyceae \\
\hline & & Order: Centrales \\
\hline & & Family: Soleniae \\
\hline & & Genus: Rhizosolenia \\
\hline & & Species: styliformis \\
\hline \multirow{5}{*}{29} & \multirow{5}{*}{ Rhizosolenia robusta } & Class: Bacillariophyceae \\
\hline & & Order: Centrales \\
\hline & & Family: Soleniae \\
\hline & & Genus: Rhizosolenia \\
\hline & & Species: robusta \\
\hline \multirow{6}{*}{30} & \multirow{6}{*}{ Rhizosolenia stolterfothii } & Division: Thallophyta \\
\hline & & Class: Bacillariophyceae \\
\hline & & Order: Centrales \\
\hline & & Family: Soleniae \\
\hline & & Genus: Rhizosolenia \\
\hline & & Species: stolterfothii \\
\hline
\end{tabular}




\begin{tabular}{|c|c|c|}
\hline \multirow{6}{*}{31} & \multirow{6}{*}{ Rhizosolenia cylindrus } & Division: Thallophyta \\
\hline & & Class: Bacillariophyceae \\
\hline & & Order: Centrales \\
\hline & & Family: Soleniae \\
\hline & & Genus: Rhizosolenia \\
\hline & & Species: cylindrus \\
\hline \multirow{6}{*}{32} & \multirow{6}{*}{ Rhizosolenia shrubsolei } & Division: Thallophyta \\
\hline & & Class: Bacillariophyceae \\
\hline & & Order: Centrales \\
\hline & & Family: Soleniae \\
\hline & & Genus: Rhizosolenia \\
\hline & & Species: shrubsolei \\
\hline \multirow{6}{*}{33} & \multirow{6}{*}{ Rhizosolenia imbricata } & Division: Thallophyta \\
\hline & & Class: Bacillariophyceae \\
\hline & & Order: Centrales \\
\hline & & Family: Soleniae \\
\hline & & Genus: Rhizosolenia \\
\hline & & Species: imbricata \\
\hline \multirow{6}{*}{34} & & Division: Thallophyta \\
\hline & & Class: Bacillariophyceae \\
\hline & & Order: Centrales \\
\hline & & Family: Soleniae \\
\hline & & Genus: Lauderia \\
\hline & & Species: annulata \\
\hline \multirow{5}{*}{35} & \multirow{5}{*}{ Bacteriastrum sp. } & Division: Thallophyta \\
\hline & & Class: Bacillariophyceae \\
\hline & & Order: Centrales \\
\hline & & Family: Chaetocereae \\
\hline & & Type: Bacteriastrum sp. \\
\hline \multirow{6}{*}{36} & \multirow{6}{*}{ Bacteriastrum delicatulum } & Division: Thallophyta \\
\hline & & Class: Bacillariophyceae \\
\hline & & Order: Centrales \\
\hline & & Family: Chaetocereae \\
\hline & & Genus: Bacteriastrum \\
\hline & & Species: delicatulum \\
\hline \multirow{6}{*}{37} & \multirow{6}{*}{ Bacteriastrum varians } & Division: Thallophyta \\
\hline & & Class: Bacillariophyceae \\
\hline & & Order: Centrales \\
\hline & & Family: Chaetocereae \\
\hline & & Genus: Bacteriastrum \\
\hline & & Species: varians \\
\hline
\end{tabular}




\begin{tabular}{|c|c|c|}
\hline \multirow{6}{*}{38} & \multirow{6}{*}{ Bacteriastrum comosum } & Division: Thallophyta \\
\hline & & Class: Bacillariophyceae \\
\hline & & Order: Centrales \\
\hline & & Family: Chaetocereae \\
\hline & & Genus: Bacteriastrum \\
\hline & & Species: comosum \\
\hline \multirow{6}{*}{39} & \multirow{6}{*}{ Bacteriastrum hyalinum } & Division: Thallophyta \\
\hline & & Class: Bacillariophyceae \\
\hline & & Order: Centrales \\
\hline & & Family: Chaetocereae \\
\hline & & Genus: Bacteriastrum \\
\hline & & Species: hyalinum \\
\hline \multirow{6}{*}{40} & \multirow{6}{*}{ Chaetoceros dydymus } & Division: Thallophyta \\
\hline & & Class: Bacillariophyceae \\
\hline & & Order: Centrales \\
\hline & & Family: Chaetocereae \\
\hline & & Genus: Chaetoceros \\
\hline & & Species: dydymus \\
\hline \multirow{6}{*}{41} & \multirow{6}{*}{ Chaetoceros curvisetus } & Division: Thallophyta \\
\hline & & Class: Bacillariophyceae \\
\hline & & Order: Centrales \\
\hline & & Family: Chaetocereae \\
\hline & & Genus: Chaetoceros \\
\hline & & Species: curvisetus \\
\hline \multirow{6}{*}{42} & \multirow{6}{*}{ Chaetoceros diversus } & Division: Thallophyta \\
\hline & & Class: Bacillariophyceae \\
\hline & & Order: Centrales \\
\hline & & Family: Chaetocereae \\
\hline & & Genus: Chaetoceros \\
\hline & & Species: diversus \\
\hline \multirow{6}{*}{43} & \multirow{6}{*}{ Chaetoceros messanensis } & Division: Thallophyta \\
\hline & & Class: Bacillariophyceae \\
\hline & & Order: Centrales \\
\hline & & Family: Chaetocereae \\
\hline & & Genus: Chaetoceros \\
\hline & & Species: messanensis \\
\hline \multirow{6}{*}{44} & \multirow{6}{*}{ Chaetoceros peruvianus } & Division: Thallophyta \\
\hline & & Class: Bacillariophyceae \\
\hline & & Order: Centrales \\
\hline & & Family: Chaetocereae \\
\hline & & Genus: Chaetoceros \\
\hline & & Species: peruvianus \\
\hline
\end{tabular}




\begin{tabular}{|c|c|c|}
\hline \multirow{6}{*}{45} & \multirow{6}{*}{ Chaetoceros eibenii } & Division: Thallophyta \\
\hline & & Class: Bacillariophyceae \\
\hline & & Order: Centrales \\
\hline & & Family: Chaetocereae \\
\hline & & Genus: Chaetoceros \\
\hline & & Species: eibenii \\
\hline \multirow{6}{*}{46} & \multirow{6}{*}{ Chaetoceros lorenzianus } & Division: Thallophyta \\
\hline & & Class: Bacillariophyceae \\
\hline & & Order: Centrales \\
\hline & & Family: Chaetocereae \\
\hline & & Genus: Chaetoceros \\
\hline & & Species: lorenzianus \\
\hline \multirow{6}{*}{47} & \multirow{6}{*}{ Chaetoceros compressus } & Division: Thallophyta \\
\hline & & Class: Bacillariophyceae \\
\hline & & Order: Centrales \\
\hline & & Family: Chaetocereae \\
\hline & & Genus: Chaetoceros \\
\hline & & Species: compressus \\
\hline \multirow{6}{*}{48} & \multirow{6}{*}{ Chaetoceros decipiens } & Division: Thallophyta \\
\hline & & Class: Bacillariophyceae \\
\hline & & Order: Centrales \\
\hline & & Family: Chaetocereae \\
\hline & & Genus: Chaetoceros \\
\hline & & Species: decipiens \\
\hline \multirow{6}{*}{49} & \multirow{6}{*}{ Chaetoceros atlanticus } & Division: Thallophyta \\
\hline & & Class: Bacillariophyceae \\
\hline & & Order: Centrales \\
\hline & & Family: Chaetocereae \\
\hline & & Genus: Chaetoceros \\
\hline & & Species: atlanticus \\
\hline \multirow{6}{*}{50} & \multirow{6}{*}{ Chaetoceros subtilis } & Division: Thallophyta \\
\hline & & Class: Bacillariophyceae \\
\hline & & Order: Centrales \\
\hline & & Family: Chaetocereae \\
\hline & & Genus: Chaetoceros \\
\hline & & Species: subtilis \\
\hline \multirow{6}{*}{51} & \multirow{6}{*}{ Chaetoceros convolutus } & Division: Thallophyta \\
\hline & & Class: Bacillariophyceae \\
\hline & & Order: Centrales \\
\hline & & Family: Chaetocereae \\
\hline & & Genus: Chaetoceros \\
\hline & & Species: convolutus \\
\hline
\end{tabular}




\begin{tabular}{|c|c|c|}
\hline \multirow{6}{*}{52} & \multirow{6}{*}{ Chaetoceros holsaticum } & Division: Thallophyta \\
\hline & & Class: Bacillariophyceae \\
\hline & & Order: Centrales \\
\hline & & Family: Chaetocereae \\
\hline & & Genus: Chaetoceros \\
\hline & & Species: holsaticum \\
\hline \multirow{6}{*}{53} & \multirow{6}{*}{ Chaetoceros gracile } & Division: Thallophyta \\
\hline & & Class: Bacillariophyceae \\
\hline & & Order: Centrales \\
\hline & & Family: Chaetocereae \\
\hline & & Genus: Chaetoceros \\
\hline & & Species: gracile \\
\hline \multirow{6}{*}{54} & \multirow{6}{*}{ Chaetoceros cinctum } & Division: Thallophyta \\
\hline & & Class: Bacillariophyceae \\
\hline & & Order: Centrales \\
\hline & & Family: Chaetocereae \\
\hline & & Genus: Chaetoceros \\
\hline & & Species: cinctum \\
\hline \multirow{6}{*}{55} & \multirow{6}{*}{ Chaetoceros affinis } & Division: Thallophyta \\
\hline & & Class: Bacillariophyceae \\
\hline & & Order: Centrales \\
\hline & & Family: Chaetocereae \\
\hline & & Genus: Chaetoceros \\
\hline & & Species: affinis \\
\hline \multirow{6}{*}{56} & \multirow{6}{*}{ Chaetoceros danicus } & Division: Thallophyta \\
\hline & & Class: Bacillariophyceae \\
\hline & & Order: Centrales \\
\hline & & Family: Chaetocereae \\
\hline & & Genus: Chaetoceros \\
\hline & & Species: danicus \\
\hline \multirow{6}{*}{57} & \multirow{6}{*}{ Chaetoceros constrictus } & Division: Thallophyta \\
\hline & & Class: Bacillariophyceae \\
\hline & & Order: Centrales \\
\hline & & Family: Chaetocereae \\
\hline & & Genus: Chaetoceros \\
\hline & & Species: constrictus \\
\hline \multirow{6}{*}{58} & \multirow{6}{*}{ Ditylum sol } & Division: Thallophyta \\
\hline & & Class: Bacillariophyceae \\
\hline & & Order: Centrales \\
\hline & & Family: Biddulphiese \\
\hline & & Genus: Ditylum \\
\hline & & Species: sol \\
\hline
\end{tabular}




\begin{tabular}{|c|c|c|}
\hline \multirow{6}{*}{59} & \multirow{6}{*}{ Ditylum brightwelli } & Division: Thallophyta \\
\hline & & Class: Bacillariophyceae \\
\hline & & Order: Centrales \\
\hline & & Family: Biddulphiese \\
\hline & & Genus: Ditylum \\
\hline & & Species: brightwelli \\
\hline \multirow{6}{*}{60} & \multirow{6}{*}{ Lithodesmium undulatum } & Division: Thallophyta \\
\hline & & Class: Bacillariophyceae \\
\hline & & Order: Centrales \\
\hline & & Family: Biddulphiese \\
\hline & & Genus: Lithodesmium \\
\hline & & Species: undulatum \\
\hline \multirow{6}{*}{61} & \multirow{6}{*}{ Triceratium favus } & Division: Thallophyta \\
\hline & & Class: Bacillariophyceae \\
\hline & & Order: Centrales \\
\hline & & Family: Biddulphiese \\
\hline & & Genus: Triceratium \\
\hline & & Species: favus \\
\hline \multirow{6}{*}{62} & \multirow{6}{*}{ Triceratium reticulatum } & Division: Thallophyta \\
\hline & & Class: Bacillariophyceae \\
\hline & & Order: Centrales \\
\hline & & Family: Biddulphiese \\
\hline & & Genus: Triceratium \\
\hline & & Species: reticulatum \\
\hline \multirow{5}{*}{63} & \multirow{5}{*}{ Triceratium sp. } & Division: Thallophyta \\
\hline & & Class: Bacillariophyceae \\
\hline & & Order: Centrales \\
\hline & & Family: Biddulphiese \\
\hline & & Type: Triceratium sp. \\
\hline \multirow{6}{*}{64} & \multirow{6}{*}{ Biddulphia sinensis } & Division: Thallophyta \\
\hline & & Class: Bacillariophyceae \\
\hline & & Order: Centrales \\
\hline & & Family: Biddulphiese \\
\hline & & Genus: Biddulphia \\
\hline & & Species: sinensis \\
\hline \multirow{6}{*}{65} & \multirow{6}{*}{ Biddulphia mobiliensis } & Division: Thallophyta \\
\hline & & Class: Bacillariophyceae \\
\hline & & Order: Centrales \\
\hline & & Family: Biddulphiese \\
\hline & & Genus: Biddulphia \\
\hline & & Species: mobiliensis \\
\hline \multirow{6}{*}{66} & \multirow{6}{*}{ Biddulphia regia } & Division: Thallophyta \\
\hline & & Class: Bacillariophyceae \\
\hline & & Order: Centrales \\
\hline & & Family: Biddulphiese \\
\hline & & Genus: Biddulphia \\
\hline & & Species: regia \\
\hline
\end{tabular}




\begin{tabular}{|c|c|c|}
\hline \multirow{6}{*}{67} & \multirow{6}{*}{ Eucampia zodiacus } & Division: Thallophyta \\
\hline & & Class: Bacillariophyceae \\
\hline & & Order: Centrales \\
\hline & & Family: Biddulphiese \\
\hline & & Genus: Eucampia \\
\hline & & Species: zodiacus \\
\hline \multirow{6}{*}{68} & \multirow{6}{*}{ Hemidiscus cuneiformis } & Division: Thallophyta \\
\hline & & Class: Bacillariophyceae \\
\hline & & Order: Centrales \\
\hline & & Family: Euodieae \\
\hline & & Genus: Hemidiscus \\
\hline & & Species: cuneiformis \\
\hline \multirow{6}{*}{69} & \multirow{6}{*}{ Climacosphenia elongata } & Division: Thallophyta \\
\hline & & Class: Bacillariophyceae \\
\hline & & Order: Pennales \\
\hline & & Family: Fragilarioideae \\
\hline & & Genus: Climacosphenia \\
\hline & & Species: elongata \\
\hline \multirow{6}{*}{70} & \multirow{6}{*}{ Fragilaria oceanica } & Division: Thallophyta \\
\hline & & Class: Bacillariophyceae \\
\hline & & Order: Pennales \\
\hline & & Family: Fragilarioideae \\
\hline & & Genus: Fragilaria \\
\hline & & Species: oceanica \\
\hline \multirow{6}{*}{71} & \multirow{6}{*}{ Rhaphoneis amphiceros } & Division: Thallophyta \\
\hline & & Class: Bacillariophyceae \\
\hline & & Order: Pennales \\
\hline & & Family: Fragilarioideae \\
\hline & & Genus: Rhaphoneis \\
\hline & & Species: amphiceros \\
\hline \multirow{6}{*}{72} & \multirow{6}{*}{ Thalassionema nitzchioides } & Division: Thallophyta \\
\hline & & Class: Bacillariophyceae \\
\hline & & Order: Pennales \\
\hline & & Family: Fragilarioideae \\
\hline & & Genus: Thalassionema \\
\hline & & Species: nitzchioides \\
\hline \multirow{5}{*}{73} & \multirow{5}{*}{ Thalassionema sp. } & Division: Thallophyta \\
\hline & & Class: Bacillariophyceae \\
\hline & & Order: Pennales \\
\hline & & Family: Fragilarioideae \\
\hline & & Type: Thalassionema sp. \\
\hline
\end{tabular}

\section{References}

1. Mitra A, Zaman S (2014) Carbon Sequestration by Coastal Floral Community. In: Mitra A, Zaman S (Eds.), Carbon Sequestration by Coastal Floral Community. Published by The Energy and Resources Institute (TERI) TERI Press, India.
2. Mitra A, Zaman S (2015) Blue Carbon Reservoir of the Blue Planet. In: Mitra A, Zaman S (Eds.), Published by Springer, India. 